\title{
Rehabilitation of spinal muscular atrophy: current consensus and future direction
}

\author{
You Gyoung Yi, ${ }^{1, \oplus}$, Hyung-lk Shin ${ }^{2} \oplus$, and Dae-Hyun Jang ${ }^{3, * \odot}$ \\ ${ }^{1}$ Department of Rehabilitation Medicine, National Traffic Injury Rehabilitation Hospital, Yangpyeong, Korea \\ 2Department of Rehabilitation, Seoul National University Hospital, Seoul National University College of Medicine, Seoul, Korea \\ ${ }^{3}$ Department of Rehabilitation, Incheon St. Mary's Hospital, College of Medicine, The Catholic University of Korea, Seoul, Korea
}

\begin{abstract}
Spinal muscular atrophy (SMA) is a neuromuscular disease that requires multidisciplinary medical care, including rehabilitation management. The emergence of a genetic therapy-based approach for SMA has markedly changed the disease course. Nonetheless, currently, updated physical therapy and rehabilitation are warranted for individuals with SMA in the era of gene therapy. In this review, we discuss the physical therapy and rehabilitation strategies currently performed for people with SMA, such as positioning and bracing, supported standing, management of musculoskeletal deformities, stretching, physical exercise training like aerobics and strengthening exercises, assistive devices, pulmonary rehabilitation, and dysphagia treatment.
\end{abstract}

Key words: Spinal muscular atrophy, Rehabilitation, Assistive device, Exercise.

\section{Introduction}

Spinal muscular atrophy (SMA) is a group of neuromuscular diseases characterized by the degeneration of the ventral horn of the spinal cord with generalized progressive muscle weakness and atrophy [1]. SMA is an autosomal recessive disorder caused by biallelic pathogenic variants in the survival motor neuron 1 gene (SMN1) on chromosome 5, resulting in decreased expression of the survival motor neuron (SMN) protein [2,3]. Patients with SMA have one or more copies of SMN2-a similar gene that produces functional SMN protein, albeit in reduced amounts [4]. The SMA disease severity varies depending on the SMN2 copy numbers $[1,4]$.

SMA is classified into four clinical subtypes based on the age at symptom onset and maximal motor milestones [1]. SMA type
1 is characterized by onset by 6 months of age and failure to sit unsupported $[3,5]$. The symptom onset of SMA type 2 is between 6 and 18 months of age, and those affected can sit independently but are non-ambulant $[1,6]$. SMA type 3 , also known as Kugelberg-Welander syndrome, is a relatively mild subtype, which develops after 18 months of age $[3,7,8]$. Most individuals with SMA type 3 acquire the ability to walk. SMA type 4 is the mildest form, which is characterized by an onset of weakness during adulthood $[1,4]$.

In 2007, the consensus statement for the standard of care for SMA was published by categorizing people with SMA into nonsitter, sitter, and walker [9]. This statement has been widely adopted by clinicians over the past decade. Nevertheless, with the recent introduction of nusinersen (Spinraza; Biogen Inc., Cambridge, MA, USA), an antisense oligonucleotide that

Received: 25 November 2020, Revised: 3 December 2020, Accepted: 3 December 2020, Published: 31 December 2020

${ }^{*}$ Corresponding author: Dae-Hyun Jang, M.D., Ph.D. (iD https://orcid.org/0000-0001-8293-084X

Department of Rehabilitation, Incheon St. Mary's Hospital, College of Medicine, The Catholic University of Korea, 56 Dongsu-ro, Bupyeong-gu, Incheon 21431, Korea.

Tel: +82-32-280-5207, Fax: +82-32-280-5040, E-mail: dhjangmd@naver.com

Conflict of interest: The authors declare that they do not have any conflicts of interest.

(c) This is an open-access article distributed under the terms of the Creative Commons Attribution Non-Commercial License (http://creativecommons.org/licenses/by-nc/4.0/) which permits unrestricted non-commercial use, distribution, and reproduction in any medium, provided the original work is properly cited.

(c) Copyright 2020 by the Korean Society of Medical Genetics and Genomics 
increases the expression of SMN2 $[2,10,11]$, there has been increasing evidence of improvements in the disease course of all SMA types [12-16]. Moreover, the recently approved SMN1 gene replacement therapy, AVXS-101 (Zolgensma; AveXis Inc., Bannockburn, IL, USA), has been reported to be effective in younger pediatric patients with SMA type 1 [17-19]. Nevertheless, with the change in the disease course in children compared with the past few years, physicians are gradually changing their perspective toward more active management of diseases $[1,12,16]$.

This paper discusses the current rehabilitation strategies performed for individuals with SMA, as well as describes the various therapeutic approaches, including positioning and bracing, supported standing, management of musculoskeletal deformities, stretching, physical exercise training including aerobics and strengthening exercises, assistive devices, pulmonary rehabilitation, and dysphagia treatment.

\section{Positioning and Bracing}

Posture maintenance, seating support, and contracture prevention are of paramount significance in people with SMA. In individuals who cannot sit without support, daily use of seating systems with postural and positioning supports are required. Seating systems and postural supports should include supine positioning with rolls, molded pillows, or wedges [1] (Fig. 1). Strollers and power wheelchairs with recline or tilt options and adapted seating systems are recommended to promote mobility and transfers [1].

Cervical bracing is often used for head support for safety and transportation because of impaired head control [1]. Cervical bracing includes a cervical jacket that provides maximal stability and the Philadelphia neck collar. Headpod can be used for proper head and neck support [20]. Spinal orthoses could be used to promote function during sitting and for postural stabilization, but there is a lack of evidence that spinal orthoses delay curve progression $[1,9,21]$.

Ankle foot orthoses, knee immobilizers, knee ankle foot orthoses, and hand splints are recommended for stretching and positioning $[1,9]$. Lower limb orthoses for ankle and knee are used for posture support in walkers. Notably, a minimal frequency of 5 times per week of applying orthoses for a minimum duration of 60 minutes to overnight has been suggested to be effective $[1,9]$.

\section{Supported Standing}

Supported standing (Fig. 2) promotes lower extremity stretching, as well as benefits bodily functions and bone and lung health. Moreover, it is essential for activating gastrointestinal function and promoting spinal alignment. It has been advocated in children who cannot walk to help them achieve a standing position [22]. The 2007 SMA standard of care recommended supported standing with bracing for children with SMA type 2 [9]. Moreover, the SMA care group recently recommended that children with SMA types 1 and 2 consider standing support. The recommended optimal frequency of supported standing is 5 to 7 times per week, with a minimum of 3 to 5 times per week for 60 minutes [1].

Townsend et al. [22] reported that the prevalence of consistent use of standing programs was 13\% in SMA type 1 and 68\%

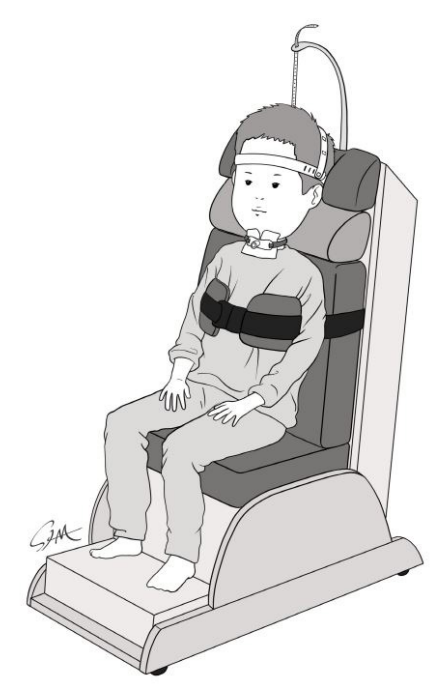

Fig. 1. Seating systems and postural supports.

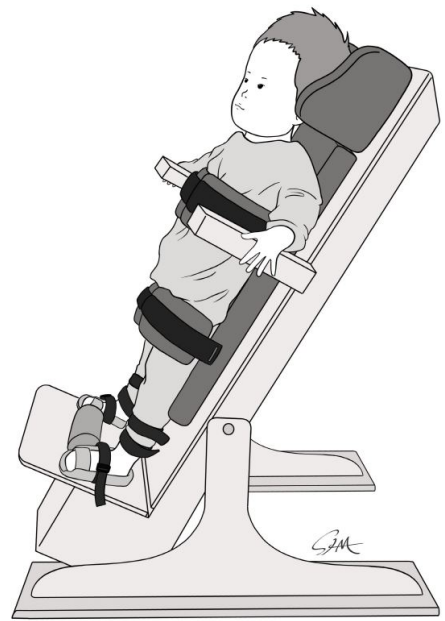

Fig. 2. Use of supine stander for supported standing with orthoses. 
in SMA type 2. It is also feasible for children who require invasive or noninvasive ventilation. Physical therapists should ensure that children with impaired head control maintain a supported standing position with protected airways and are closely supervised when standing [22]. Lower extremity orthoses, such as ankle-foot orthoses and knee braces, can be applied to optimize joint alignment and prevent excessive valgus force on the knee and to optimize hip joint alignment by placing the hip joint in a slightly abducted position [22].

\section{Management of Musculoskeletal Deformities including Scoliosis and Hip Dislocation}

Scoliosis is highly prevalent in children with SMA 1 and 2, with an incidence of 60 to $90 \%$ and an initial presentation during early childhood $[23,24]$. The spinal curves consistently progress through childhood in these individuals. In addition, a variable degree of thoracic kyphosis is prevalent in children with SMA. For positioning, thoracolumbosacral orthosis and static thoracic bracing can be used with incorporated modifications for respiratory support, including abdominal cutouts [1]. However, bracing cannot prevent the progression of spinal deformity. Spinal orthosis is recommended if the child has Cobb's angle of more than 20 degrees with hypotonic trunk [1]. Distortion of the rib cage related to scoliosis is associated with respiratory disease, and pelvic obliquity associated with scoliosis affects the ability to maintain balance in the sitting position [25]. Furthermore, hip dislocation or subluxation is common in people with SMA [26]. Hip instability with associated pelvic obliquity is prevalent in individuals with SMA types 1 and 2 and less common in type 3 patients [26]. Surgical treatment is indicated for unilateral and bilateral hip instabilities only in patients with significant pain [1].

\section{Stretching Exercises}

Stretching includes the active-assistive and passive skills, and use of orthoses, serial casting, and supported standing for positioning [7,9,27-29]. Upper and lower limb orthoses are used to increase the range of motion and promote function $[1,30]$. Stretching should be performed directly or under the supervision of a physical therapist or occupational therapist [1]. In addition, parents and caregivers should be trained to perform a daily stretching program $[1,29]$. Each stretching session should focus on joints, rehabilitation purposes, and specific patient needs to achieve maximum benefits $[1,29]$. Notably, a minimum frequency of 3-5 times a week and optimal frequency of 5-7 times a week of stretching exercise is recommended in individuals who cannot walk. Similarly, a minimum frequency of 2-3 times a week and optimum frequency of 3-5 times a week is recommended in walkers.

\section{Physical Exercise Training including Aerobics and Strengthening Exercises}

Whether physical exercise training is beneficial or detrimental for patients with SMA has been a debatable topic $[7,8]$. Even though several animal studies of physical exercise training have evidenced positive effects regarding improving the motor function and stimulating SMN expression, there is limited clinical evidence [31-33]. However, the recent approval of genetic treatment with Spinraza and Zolgensma might enable active physical exercise to be effective.

\section{Current clinical evidence}

Active physical exercise training of patients with SMA was considered to be likely harmful or not helpful because of muscle fatigue-induced weakness and the lack of clinical evidence [3335]. To date, approximately 10 clinical studies have reported the effectiveness of physical exercise in patients with SMA $[33,36]$. Most of these studies involved small sample sizes (less than 10 patients per cohort) and were non-randomized controlled trials except for one study [37]. The exercise regimen was aerobics or strengthening exercises, and patients with SMA type 2 or 3 were included. Aerobic exercise training aims to increase exercise capacity and endurance, and strengthening might improve muscle strength and functional performance. Most reports have demonstrated the feasibility and safety of physical exercise in patients with SMA and revealed no increase in plasma creatinine kinase levels, fatigue or pain, and adverse events. Moreover, studies revealed some increase in physical performance, functional capacity, and muscle strength. Based on these observations of clinical improvement and safety, submaximal strengthening and moderate-intensity aerobic exercise training have been recommended in practice [28]. However, the therapeutic efficacy of physical exercise is inconclusive because of the small sample size, poor methodological designs, and inadequate compliance of study patients [33]. Some participants were excluded from the study because of fatigue or insufficient cooperation, and the program designs were modified per individual needs during the study $[38,39]$. A recent Cochrane review concluded that it is uncertain whether physical exercise is beneficial or harmful in patients with SMA and requested more well-designed and pow- 
ered studies to assess this aspect [8].

\section{Animal studies}

Grondard et al. [40] first reported the neuroprotective effects of physical exercise through running-based training in severe type SMA-like mice in 2005. The authors demonstrated that forced wheel running improved motor function and survival time and reduced neuronal loss in the ventral horn of the spinal cord. After the first report, by using the same experimental designs, they exhibited that physical exercise enhanced the maturation of the motor unit and the expression of the gene encoding the NMDA receptor [31]. Overall, they suggested physical exercise to be a potential therapeutic approach in patients with SMA. Besides severe type SMA-like mice, Chali et al. [32] examined two long-term physical exercises, namely the 10-months high-intensity swimming or low-intensity running training, in mild type SMA-like mice. The authors reported that both exercise paradigms improved motor function, muscle fatigability, and neuroprotection of motor neurons and prevented neuromuscular junction and muscle phenotype alterations. Furthermore, they demonstrated that exercise-induced motor neuron enhancements were independent of SMN expression and related to the specific characteristics of each exercise, indicating that the programs and protocols of physical exercise training are crucial. Recently, $\mathrm{Ng}$ et al. [41] reported that exercise activated AMP-activated protein kinase, p38 mitogen-activated protein kinase, and peroxisome proliferator-activated receptor- $\gamma$ coactivator $1 \alpha$, and altered the molecules involved in SMN transcription. The authors suggested that these changes observed after physical exercise indicated an increase in full-length SMN expression [41].

\section{Future direction}

Although there is insufficient clinical evidence currently, physical exercise training is cost-effective and can be applied at home without serious adverse events in patients with SMA. Moreover, based on the functional and neurologic status improvement in patients with all types of SMA with the recent genetic treatment with Spinraza and Zolgensma, a more active physical exercise program could be applicable. A recent randomized controlled study revealed that high-intensity training improved fitness in patients with spinal and bulbar muscular atrophy (SBMA) [42]. Nevertheless, SMA has a different genetic cause than SMBA, but because SMBA is a common motor neuron disease and similar in pattern to mild type SMA, this report suggested that physical exercise can be safe and effective in patients with chronic motor neuron disease. Nonetheless, further studies regarding the optimal dosing and effective physical exercise program designs are warranted in patients with genetically-advanced stages of SMA.

\section{Assistive Devices}

In nonsitters, gait training devices and mobility devices can be used for supported ambulation, and mobile arm support can assist the upper extremity function [1]. Eye-tracking devices can be used for communication. Moreover, strollers, power wheelchairs with recline or flat, lift, and adapted seating could help those with SMA [1]. In addition, a body support walker is feasible for those who cannot ambulate.

The use of slings (Fig. 3) or mobile arm supports can augment functional abilities and active range of motion $[7,9,21,28,30]$. The use of linear elastic devices to balance the effects of gravity in various directions can help people with proximal weakness and improve the control of distal function [9]. Mobile arm supports [43], such as Armon Ayura and JAECO WREX Supports [44] can be attached to most wheelchairs and mobility seating systems.

In sitters with sufficient strength, reciprocal gait orthoses or lightweight ischial weight-bearing knee ankle foot orthoses should be considered for standing or supported ambulation with a walker [9]. Furthermore, adaptive equipment and assistive technology should be considered to promote independent work and play [9]. Mobile arm support and electric wheelchairs with appropriate seating systems are also recommended $[9,11,30]$. Moreover, power assist wheels can be considered in sitters with adequate strength $[1,45]$.

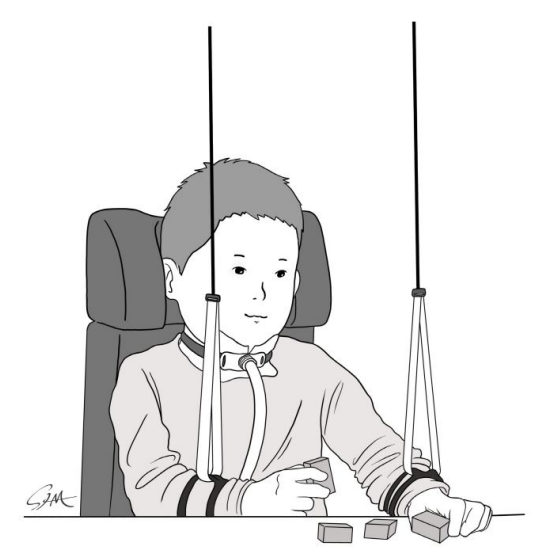

Fig. 3. Slings that helps functional abilities and active range of motion in individuals with spinal muscular atrophy. 


\section{Pulmonary Rehabilitation}

Depending on the type of SMA and the degree of muscle function loss, SMA often affects the respiratory system. Natural history studies revealed that $61 \%$ of individuals with SMA type 1 required at least one intubation within 1 year after birth [46], and that 100\% of children with SMA type 1 older than 12 months required ventilatory support owing to respiratory failure [47]. Respiratory support options include noninvasive ventilation with bilevel positive airway pressure or a mechanical ventilator and invasive ventilation with a tracheotomy. In SMA type 2 and non-ambulant type 3 , it has been reported that lung function declines differently with a common leveling after age 13 years [16].

Mobilization of secretions involves manual or mechanical chest physiotherapy with postural drainage $[9,48]$. Prone positioning might assist postural drainage, as illustrated in Fig. 4. Because the functional residual capacity in SMA is smallest in the Trendelenburg position, positioning in the Trendelenburg position during respiratory compromise might benefit diaphragmatic function [48]. For secretion clearance, performing airway clearance twice a day is recommended in SMA type 1. Children with SMA type 2 are recommended to perform airway clearance as needed. Manual cough assistance and mechanical insufflation-exsufflation with the CoughAssist should be used as often as needed because mechanical insufflation-exsufflation has been noted to improve vital capacity in individuals with advanced neuromuscular disorders [49]. Children with SMA type 3 might need airway clearance postoperatively and during serious illnesses [16].

Most individuals treated with nusinersen were observed to need assisted ventilation, which did not deteriorate as expected in SMA type 1 or improve [14]. It has been reported that respiratory muscle performance was significantly better in children with SMA type 2 with 6 injections of nusinersen than the agematched SMA type 2 historical controls [15].

\section{Swallowing and Nutrition}

Poor head control, limited mouth opening, and difficulties conveying food to the mouth is often noted in individuals with SMA that hinders their swallowing initiation. Moreover, chewing difficulties, oral hypersensitivity, tongue movement abnormalities, reduced range of mandibular motion, and increased fatigue of masticatory muscles affect the oral phase of swallowing in these individuals [7]. The contracture of the masseter muscles

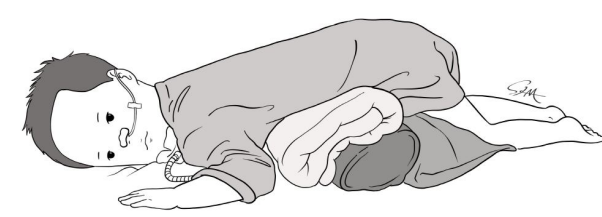

Fig. 4. Prone positioning for postural drainage of secretion.

often develops in patients by 1 year of age. This issue limits the opportunity for oral feeding in children with SMA type 1. Chewing difficulties and fatigue with eating are frequently observed in individuals with SMA type 2 [9].

Bulbar dysfunction can cause aspiration and pulmonary infections. Moreover, swallowing function has been reported to deteriorate in individuals with SMA type 1 at approximately 6 months of age, with the initiation of tube feeding at the median age of 6 months [50]. Therefore, physicians suggest videofluoroscopic swallowing study when there are clinical signs of dysphagia, such as insufficient sucking, wet voice, and pulmonary infection. In individuals with neuromuscular disease who have a tracheostomy, oral feeding challenges at a starting low dose of 0.1-0.2 $\mathrm{mL}$ with gradual increase might be attempted regardless of aspiration findings [51].

\section{Conclusion}

We are currently moving toward a new era in SMA management thanks to the advent of genetic treatment. This treatment approach can be considered as a transition to chronic and mild types from progressive neurodegenerative characteristics. Therefore, it is imperative to provide long-term comprehensive medical care involving a multidisciplinary team. Notably, rehabilitation strategies, including positioning and bracing, supported standing, management of musculoskeletal deformities, stretching, physical exercise training including aerobics and strengthening exercises, assistive devices, pulmonary rehabilitation, and dysphagia treatment can improve the motor function, performance, activities of daily life, and quality of life of patients with SMA. Therefore, it is crucial to begin these therapeutic strategies earlier in the disease course. Nonetheless, further studies are required to evaluate the effectiveness of these strategies.

\section{Acknowledgements}

We would like to thank individuals with SMA who visited the 
clinic and inspired the authors.

\section{References}

1. Mercuri E, Finkel RS, Muntoni F, Wirth B, Montes J, Main M, et al.; SMA Care Group. Diagnosis and management of spinal muscular atrophy: part 1: recommendations for diagnosis, rehabilitation, orthopedic and nutritional care. Neuromuscul Disord 2018;28:103-15.

2. Mercuri E, Darras BT, Chiriboga CA, Day JW, Campbell C, Connolly AM, et al.; CHERISH Study Group. Nusinersen versus Sham control in later-onset spinal muscular atrophy. N Engl J Med 2018;378:625-35.

3. Lefebvre $S$, Bürglen $L$, Reboullet $S$, Clermont 0 , Burlet $P$, Viollet $L$, et al. Identification and characterization of a spinal muscular atrophydetermining gene. Cell 1995;80:155-65.

4. Czech C, Tang W, Bugawan T, Mano C, Horn C, Iglesias VA, et al. Biomarker for spinal muscular atrophy: expression of SMN in peripheral blood of SMA patients and healthy controls. PLoS One 2015;10:e0139950.

5. Kolb SJ, Coffey CS, Yankey JW, Krosschell K, Arnold WD, Rutkove SB, et al.; NeuroNEXT Clinical Trial Network on behalf of the NN101 SMA Biomarker Investigators. Natural history of infantile-onset spinal muscular atrophy. Ann Neurol 2017;82:883-91.

6. Rudnik-Schöneborn S, Hausmanowa-Petrusewicz I, Borkowska J, Zerres K. The predictive value of achieved motor milestones assessed in 441 patients with infantile spinal muscular atrophy types II and III. Eur Neurol 2001;45:174-81.

7. Castro D, lannaccone ST. Spinal muscular atrophy: therapeutic strategies. Curr Treat Options Neurol 2014;16:316.

8. Rathore FA, Afridi A. Does physical exercise training improve functional performance in type 3 spinal muscular atrophy? A Cochrane Review summary with commentary. Dev Med Child Neurol 2020;62:1014-6.

9. Wang $\mathrm{CH}_{\text {, Finkel }} \mathrm{RS}$, Bertini ES, Schroth $\mathrm{M}$, Simonds $A$, Wong $B$, et al.; Participants of the International Conference on SMA Standard of Care. Consensus statement for standard of care in spinal muscular atrophy. J Child Neurol 2007;22:1027-49.

10. Finkel RS, Mercuri E, Darras BT, Connolly AM, Kuntz NL, Kirschner J, et al.; ENDEAR Study Group. Nusinersen versus Sham control in infantile-onset spinal muscular atrophy. N Engl J Med 2017;377:1723-32.

11. Hully M, Barnerias C, Chabalier D, Le Guen S, Germa V, Deladriere $E_{1}$ et al. Palliative care in SMA type 1: a prospective multicenter French study based on parents' reports. Front Pediatr 2020;8:4.

12. Montes J, Dunaway Young S, Mazzone ES, Pasternak A, Glanzman AM, Finkel RS, et al.; CS2 and CS12 Study Groups. Nusinersen improves walking distance and reduces fatigue in later-onset spinal muscular atrophy. Muscle Nerve 2019;60:409-14.
13. Maggi L, Bello L, Bonanno S, Govoni A, Caponnetto C, Passamano $L$, et al. Nusinersen safety and effects on motor function in adult spinal muscular atrophy type 2 and 3. J Neurol Neurosurg Psychiatry 2020;91:1166-74.

14. Lavie $M$, Diamant $N$, Cahal $M$, Sadot $E$, Be'er $M$, Fattal-Valevski $A$, et al. Nusinersen for spinal muscular atrophy type 1: real-world respiratory experience. Pediatr Pulmonol 2021;56:291-8.

15. Gómez-García de la Banda M, Amaddeo A, Khirani S, Pruvost $S$, Barnerias C, Dabaj I, et al. Assessment of respiratory muscles and motor function in children with SMA treated by nusinersen. Pediatr Pulmonol 2021;56:299-306.

16. Trucco F, Ridout $D$, Scoto $M$, Coratti $G$, Main ML, Lofra RM, et al.; international SMA consortium (iSMAc). Respiratory trajectories in type 2 and non-ambulant 3 Spinal muscular atrophy in the iSMAC cohort study. Neurology 2020, in press.

17. Stevens D, Claborn MK, Gildon BL, Kessler TL, Walker C. Onasemnogene abeparvovec-xioi: gene therapy for spinal muscular atrophy. Ann Pharmacother 2020;54:1001-9.

18. Waldrop MA, Karingada C, Storey MA, Powers $B$, lammarino MA, Miller NF, et al. Gene therapy for spinal muscular atrophy: safety and early outcomes. Pediatrics 2020;146:e20200729.

19. Vu-Han TL, Weiß C, Pumberger M. Novel therapies for spinal muscular atrophy are likely changing the patient phenotype. Spine J 2020;20:1893-8.

20. Brown JE, Thompson M, Brizzolara K. Head control changes after headpod use in children with poor head control: a feasibility study. Pediatr Phys Ther 2018;30:142-8.

21. Garg S. Management of scoliosis in patients with Duchenne muscular dystrophy and spinal muscular atrophy: a literature review. J Pediatr Rehabil Med 2016;9:23-9.

22. Townsend EL, Simeone SD, Krosschell KJ, Zhang RZ, Swoboda KJ; Project Cure SMA Investigator's Network. Stander use in spinal muscular atrophy: results from a large natural history database. Pediatr Phys Ther 2020;32:235-41.

23. Sumner CJ, Fischbeck KH. Spinal muscular atrophy. In: Gilman S, ed. Neurobiology of disease. Cambridge: Academic Press, 2007;501-11.

24. Mercuri E, Bertini E, lannaccone ST. Childhood spinal muscular atrophy: controversies and challenges. Lancet Neurol 2012;11:443-52.

25. Stępień A, Mazurkiewicz Ł, Maślanko K, Rekowski W, Jędrzejowska M. Cervical rotation, chest deformity and pelvic obliquity in patients with spinal muscular atrophy. BMC Musculoskelet Disord 2020;21:726.

26. Sporer SM, Smith BG. Hip dislocation in patients with spinal muscular atrophy. J Pediatr Orthop 2003;23:10-4.

27. Montes J, Young SD, Mazzone E, Main M. First international workshop on rehabilitation management and clinical outcome measures for spinal muscular atrophy. Neuromuscul Disord 2017;27:964-9.

28. Sheikh AM, Vissing J. Exercise therapy for muscle and lower motor 
neuron diseases. Acta Myol 2019;38:215-32.

29. Dunaway S, Montes J, McDermott MP, Martens W, Neisen A, Glanzman AM, et al. Physical therapy services received by individuals with spinal muscular atrophy (SMA). J Pediatr Rehabil Med 2016;9:35-44.

30. Rahman T, Sample W, Seliktar R, Scavina MT, Clark AL, Moran K, et al. Design and testing of a functional arm orthosis in patients with neuromuscular diseases. IEEE Trans Neural Syst Rehabil Eng 2007;15:244-51.

31. Biondi $\mathrm{O}$, Grondard $C_{\text {, Lécolle }} \mathrm{S}_{\text {, Deforges }} \mathrm{S}_{\text {, Pariset }} \mathrm{C}_{\text {, Lopes }}$, et al. Exercise-induced activation of NMDA receptor promotes motor unit development and survival in a type 2 spinal muscular atrophy model mouse. J Neurosci 2008;28:953-62.

32. Chali $F$, Desseille $C$, Houdebine $L$, Benoit $E$, Rouquet $T$, Bariohay $B$, et al. Long-term exercise-specific neuroprotection in spinal muscular atrophy-like mice. J Physiol 2016;594:1931-52.

33. Bartels B, Montes J, van der Pol WL, de Groot JF. Physical exercise training for type 3 spinal muscular atrophy. Cochrane Database Syst Rev 2019;3:CD012120.

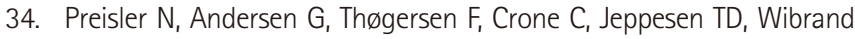
$F_{1}$ et al. Effect of aerobic training in patients with spinal and bulbar muscular atrophy (Kennedy disease). Neurology 2009;72:317-23.

35. Lewelt A, Krosschell KJ, Stoddard GJ, Weng C, Xue M, Marcus RL, et al. Resistance strength training exercise in children with spinal muscular atrophy. Muscle Nerve 2015;52:559-67.

36. Ng SY, Manta A, Ljubicic V. Exercise biology of neuromuscular disorders. Appl Physiol Nutr Metab 2018;43:1194-206.

37. Montes J, Garber CE, Kramer SS, Montgomery MJ, Dunaway S, KamilRosenberg S, et al. Single-blind, randomized, controlled clinical trial of exercise in ambulatory spinal muscular atrophy: why are the results negative? J Neuromuscul Dis 2015;2:463-70.

38. Madsen KL, Hansen RS, Preisler N, Thøgersen F, Berthelsen MP, Vissing J. Training improves oxidative capacity, but not function, in spinal muscular atrophy type III. Muscle Nerve 2015;52:240-4.

39. Bora G, Subaşı-Yıldız Ş, Yeşbek-Kaymaz A, Bulut N, Alemdaroğlu İ, Tunca-Yılmaz Ö, et al. Effects of arm cycling exercise in spinal muscular atrophy type II patients: a pilot study. J Child Neurol 2018;33:209-15.
40. Grondard C, Biondi O, Armand AS, Lécolle S, Della Gaspera B, Pariset

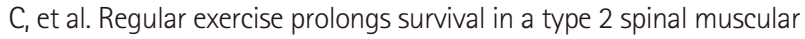
atrophy model mouse. J Neurosci 2005;25:7615-22.

41. Ng SY, Mikhail A, Ljubicic V. Mechanisms of exercise-induced survival motor neuron expression in the skeletal muscle of spinal muscular atrophy-like mice. J Physiol 2019;597:4757-78.

42. Heje $K$, Andersen G, Buch A, Andersen H, Vissing J. High-intensity training in patients with spinal and bulbar muscular atrophy. J Neurol 2019;266:1693-7.

43. Essers J, Murgia A, Peters A, Meijer K. Daily life benefits and usage characteristics of dynamic arm supports in subjects with neuromuscular disorders. Sensors (Basel) 2020;20:4864.

44. Essers JMN, Murgia A, Peters AA, Janssen MMHP, Meijer K. Recommendations for studies on dynamic arm support devices in people with neuromuscular disorders: a scoping review with expert-based discussion. Disabil Rehabil Assist Technol 2020, in press.

45. Jones MA, McEwen IR, Hansen L. Use of power mobility for a young child with spinal muscular atrophy. Phys Ther 2003;83:253-62.

46. Bach JR, Baird JS, Plosky D, Navado J, Weaver B. Spinal muscular atrophy type 1: management and outcomes. Pediatr Pulmonol 2002;34:16-22.

47. Finkel RS, McDermott MP, Kaufmann P, Darras BT, Chung WK, Sproule DM, et al. Observational study of spinal muscular atrophy type I and implications for clinical trials. Neurology 2014;83:810-7.

48. Schroth MK. Special considerations in the respiratory management of spinal muscular atrophy. Pediatrics 2009;123 Suppl 4:S245-9.

49. Stehling $F$, Bouikidis A, Schara U, Mellies U. Mechanical insufflation/ exsufflation improves vital capacity in neuromuscular disorders. Chron Respir Dis 2015;12:31-5.

50. Choi YA, Suh DI, Chae JH, Shin HI. Trajectory of change in the swallowing status in spinal muscular atrophy type I. Int J Pediatr Otorhinolaryngol 2020;130:109818.

51. Yi YG, Oh BM, Yang $S$, Shin HI. Oral feeding challenges in children with tracheostomy can improve feeding outcomes, even with the finding of aspiration. Front Pediatr 2019;7:362. 